\title{
Correction to: A Simple Kinetic Model for the Phase Transition of the van der Waals Fluid
}

\section{Shigeru Takata ${ }^{1}$ (D) Takashi Noguchi ${ }^{1}$}

Published online: 10 October 2021

(c) Springer Science+Business Media, LLC, part of Springer Nature 2021

\section{Correction to: J Stat Phys (2018) 172: 880-903 https://doi.org/10.1007/s10955-018-2068-z}

In the original article, $\tilde{x}$ in (69a) should read $x$ and the last line of (70a) should be replaced by

$$
\begin{aligned}
& -K \delta_{h}^{2}\left[\delta_{h}^{2}[\chi](x, y)\right](x, y)-K \delta_{h x}[\chi](x, y) \delta_{h x}\left[\delta_{h}^{2}[\chi](x, y)\right](x, y) \\
& -K \delta_{h y}[\chi](x, y) \delta_{h y}\left[\delta_{h}^{2}[\chi](x, y)\right](x, y) .
\end{aligned}
$$

The error in (70a) affects the numerical results for 2D cases presented in Figs. 5, 6, and 7b, though their characteristic features remain unchanged. The corrected Figs. 5, 6, and 7b are shown below.

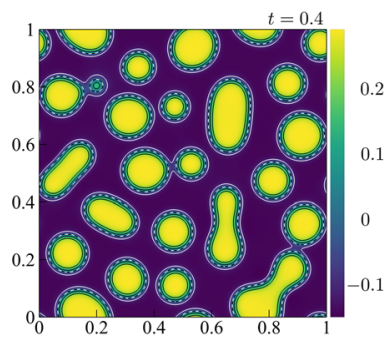

(a) $c=3.8, \chi_{\mathrm{av}}=17 / 60$

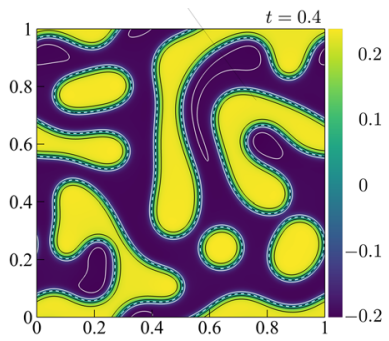

(b) $c=3.8, \chi_{\mathrm{av}}=1 / 3$

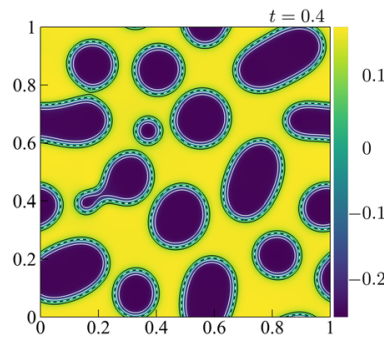

(c) $c=3.8, \chi_{\mathrm{av}}=23 / 60$

Fig. 5 Contour plots of the rescaled density $\chi$ on the $x y$-plane: two coexisting phases at the instance $t=0.400$ induced by an Gaussian noise (with the standard deviation of 0.001) disturbance of an initial uniform state. The scale number in the legend indicates the value of $\chi-\chi_{\mathrm{av}}$. The contours are drawn with the interval of 0.1 . The contour of $\chi=\chi_{\mathrm{av}}$ is drawn by a dotted line, while other contours by solid lines

The original article can be found online at https://doi.org/10.1007/s10955-018-2068-z.

\footnotetext{
$\bowtie$ Shigeru Takata

takata.shigeru.4a@kyoto-u.ac.jp

1 Department of Aeronautics and Astronautics, Kyoto University, Kyoto 615-8540, Japan
} 


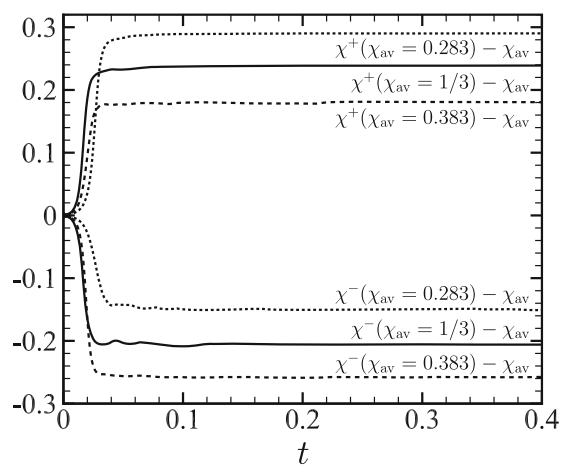

(a)

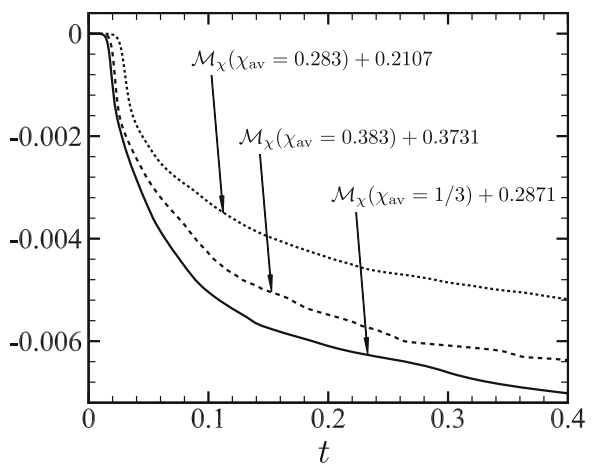

(b)

Fig. 6 Time evolution of the maximum/minimum of $\chi$, say $\chi^{+}$and $\chi^{-}$, and that of the system total free energy $M_{\chi}$ in two dimensional cases. a $\chi^{ \pm}$vs. $t$, b $M_{\chi}$ vs. $t$. Two parameters $K$ and $c$ are commonly set as $K=4.3976 \times 10^{-5}$ and $c=3.8$, while the values of $\chi_{\mathrm{av}}$ are shown in the figure. The initial values of $M_{\chi}$ are $-0.2107,-0.2871$, and -0.3731 for $\chi_{\mathrm{av}}=0.283(=17 / 60), 1 / 3$, and $0.383(=23 / 60)$, respectively

Fig. 7 Grid dependence of the results for the case $c=3.8$, $\chi_{\mathrm{av}}=1 / 3$, and $K=4.3976 \times 10^{-5}$. b The difference $\Delta$ at $t=0.2$ between the results with standard and double-size coarse grids in the $2 \mathrm{D}$ simulation. The three types of hatched area, namely the upward hatched area with wide interval, the downward hatched area, and the upward hatched area with narrow interval, represent the area where $0.003<\Delta<0.01$, $0.01<\Delta<0.03$, and $0.03<\Delta$. The scale in the legend shows $\chi-\chi_{\mathrm{av}}$

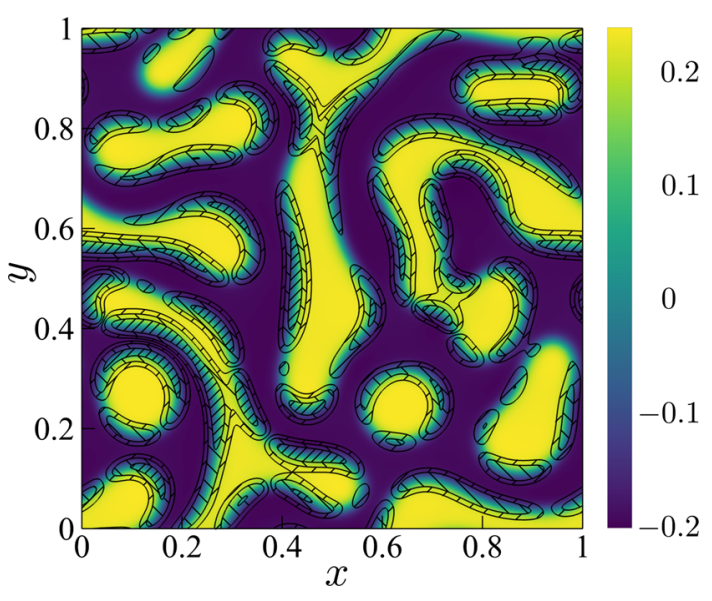

The authors thank Dr. Masanari Hattori for pointing out the error in (70a) and for his help in preparing new figures.

Publisher's Note Springer Nature remains neutral with regard to jurisdictional claims in published maps and institutional affiliations. 